\title{
Combinatorial modular pathway engineering for guanosine 5'-diphosphate-L-fucose production in recombinant Escherichia coli
}

\section{Li Wan}

Jiangnan University

Yingying Zhu

Jiangnan University

\section{Wen Li}

Jiangnan University

Wenli Zhang

Jiangnan University

Wanmeng Mu ( $\nabla$ wmmu@jiangnan.edu.cn )

Jiangnan University

\section{Research}

Keywords: GDP-L-fucose, modular pathway engineering, cofactor regeneration, the de novo pathway

Posted Date: January 14th, 2020

DOI: https://doi.org/10.21203/rs.2.20808/v1

License: (c) (1) This work is licensed under a Creative Commons Attribution 4.0 International License. Read Full License 


\section{Abstract}

Background: Guanosine 5'-diphosphate (GDP)-L-fucose is a vital nucleotide sugar involved in the synthesis of fucosylated oligosaccharides, such as fucosylated human milk oligosaccharides, which play important roles in physiological and pathological processes.

Results: In this study, a combinatorial modular pathway engineering strategy was implemented to efficiently increase the intracellular titers of GDP-L-fucose in engineered Escherichia coli. The de novo GDP-L-fucose synthesis pathway was partitioned into two modules and fine-tuned in both transcriptional and translational levels, which remarkably improved the GDP-L-fucose production. In addition, the gene encoding the UDP-glucose lipid carrier transferase (WcaJ) was inactivated to eliminate the competing metabolite pathway from GDP-L-fucose to colanic acid. Furthermore, cofactors regeneration was underpinned to promote biocatalysis. Taken together, the final engineered strain EWL37, which could achieve the titers of $18.33 \mathrm{mg} / \mathrm{L}$ in shake-flask cultivation, was able to produce $106.21 \mathrm{mg} / \mathrm{L}(4.28 \mathrm{mg} / \mathrm{g}$ DCW) GDP-L-fucose through fed-batch cultivation.

Conclusion: To date, this is the first utilization of a modular pathway optimization approach to increase the production potential of the de novo synthesized GDP-L-fucose. In general, this study manifests that via combinatorial modular pathway engineering, the GDP-L-fucose biosynthesis can be significantly improved.

\section{Background}

Guanosine 5'-diphosphate (GDP)-L-fucose, a nucleotide sugar, is found in various organisms as a glycosyl donor and metabolic intermediate, and is an imperative substance for the regulation of metabolism [1]. GDP-L-fucose is primarily harnessed as an important glycosyl donor for protein glycosylation and as a precursor for the synthesis of other sugar nucleotides in eukaryotes. In addition, it is mainly involved in the biotransformation of fucosylated oligosaccharides in prokaryotes [2].

Human milk oligosaccharides (HMOs), one of the most abundant solid components in human milk, display enormous promise for infant development due to their biological functions such as improvement of intestinal microbiota, inhibition of pathogen adhesion, modulation of immune responses, and promotion of brain development [3-4]. Among HMOs, the proportion of fucosylated oligosaccharides, which require GDP-L-fucose as fucose donor and form a-L-fucosyl linkages with lactose or other more complicated nascent oligosaccharides, is approximately 50\% [5-6]. However, large-scale production of fucosylated HMOs can be an arduous task due to the limitation in the availability of GDP-L-fucose. As an indispensable precursor in the biosynthesis of fucosylated HMOs, efficient production of GDP-L-fucose is very important.

GDP-L-fucose is synthesized in vivo through the de novo and the salvage pathway. The de novo pathway, existing in both prokaryotes and eukaryotes, transforms fructose-6-phosphate to GDP-D-mannose via three reactions carried out by three enzymes, mannose-6-phosphate isomerase (ManA, E.C. 5.3.1.8), 
phosphomannomutase (ManB, E.C. 5.4.2.8) and a-D-mannose-1-phosphate guanyltransferase (ManC, E.C. 2.7.7.22). Further conversion of GDP-D-mannose to GDP-L-fucose through three additional enzymatic reactions is controlled by GDP-D-mannose-4,6-dehydratase (Gmd, E.C. 4.2.1.47) and GDP-L-fucose synthetase (WcaG, E.C. 1.1.1.271) [7]. Specifically, guanosine 5'-triphosphate (GTP) and NADPH are required as cofactors into the reaction catalyzed by ManC and WcaG, respectively. The de novo pathway for the biosynthesis of GDP-L-fucose in Escherichia coli is illustrated in Fig. 1. In the salvage pathway, the bifunctional enzyme, fucokinase (EC 2.7.1.52)/fucose-1-phosphate guanylyltransferase (EC 2.7.7.30) (Fkp) from Bacteroides fragilis has been successfully introduced into E. coli, and converts exogenous fucose into GDP-L-fucose by a two-step enzymatic reaction [8-9]. Particularly, adenosine 5'-triphosphate (ATP) and GTP are two necessary cofactors for this reaction.

Thus far, several recombinant microorganisms have been developed for the biosynthesis of GDP-Lfucose [9]. Lactococcus lactis subsp. cremoris NZ9000 was utilized to express the four key enzymes (ManB, ManC,Gmd and WcaG) and produced GDP-L-fucose via one-pot synthesis strategy using mannose-6-phosphate as substrate. Although the conversion efficiency of the substrate was relatively high, the regeneration of GTP may be a big challenge for further production of GDP-L-fucose through a multiple-enzymatic cascade. Besides, both the de novo pathway and salvage pathway of GDP-L-fucose production have been constructed into recombinant Saccharomyces cerevisiae but with relatively low yield [10-12]. Furthermore, Corynebacterium glutamicum has also been engineered as a host strain for GDP-L-fucose biosynthesis by co-expressing four enzymes involved in the de novo pathway, as mentioned above [13]. Seo et al. [14-17] have performed several studies to increase the production of GDP-L-fucose based on the de novo pathway in engineered E. coli. Via optimizing carbon source, overexpressing crucial enzymes, reinforcing the regeneration of NADPH or GTP as cofactors, and modulating the fed-batch fermentation mode, the titer of GDP-L-fucose was significantly improved from $55.2 \mathrm{mg} / \mathrm{L}$ to $305.5 \mathrm{mg} / \mathrm{L}$ and the specific GDP-L-fucose content accumulated to $3.5 \mathrm{mg} / \mathrm{g}$ cell. In addition, the salvage pathway of GDP-L-fucose production has also been introduced into E. coli, and GTP regeneration was enhanced via overexpressing enzymes involved in the GTP biosynthesis pathway [7]. Baumgartner et al. integrated recombinant genes of the de novo and salvage pathway into the chromosome of E. coli JM109 for GDP-L-fucose production and enhanced the specific intracellular GDPL-fucose concentrations up to $5 \mathrm{mg} / \mathrm{g}$ dry cell wight (DCW) [18]. Furthermore, the up-regulation of the RcsA, a positive transcriptional regulator of colanic acid synthesis pathway gene cluster, can strengthen the GDP-L-fucose production in E. coli [19].

Nevertheless, previous studies have merely considered the individual variables and modulated the whole metabolic pathway in a linear mode, which may lead to metabolic burden and flux imbalances and can hardly achieve global superior yield [20-21]. To this end, combinatorial modular pathway engineering provides a pivotal approach to optimize the metabolic pathway and create optimal microbial cell factories for GDP-L-fucose production.

In this work, four key enzymes involved in the de novo pathway were partitioned into two modules and their expression was synergistically tuned at the transcription and translation levels to balance metabolic 
flux in E. coli BL21 (DE3). Besides, in the cofactor engineering module, the regeneration of NADPH and GTP was also considered via overexpressing key enzymes in the pentose phosphate pathway (PPP) and the guanosine nucleotides biosynthetic pathway. Moreover, the intracellular concentration of the desired product was remarkably increased by using the CRISPR/Cas9 system to inactivate the UDP-glucose lipid carrier transferase gene (wcaJ). Hereafter, fed-batch fermentation was conducted in order to further explore the production potential of the optimal solution. Taken together, this is the first time to implement combinatorial modular pathway engineering to modulate the de novo pathway for GDP-L-fucose production in recombinant E. coli. Strategies carried out in this study could be applicable for the construction of microorganisms as versatile microbial cell factories for the biosynthesis of other industrially high-valued metabolites.

\section{Results And Discussion}

Transcriptional fine tuning to improve GDP-L-fucose production

The regulation of the dosage of specific genes is a common strategy in metabolic engineering to modulate the number of enzyme copies in the host strain cell. A commonly employed approach is the combination of plasmids with different gene copy numbers to modulate the metabolic flux of different pathway modules in E. coli and other microorganisms [22-24]. The metabolic pathway leading to GDP-Lfucose is a native pathway in E. coli, but the productivity is extremely low. As an efficient method to regulate the metabolic pathway, combinatorial optimization of gene expression levels based on gene copy number was applied.

Given that GDP-D-mannose is a pivotal precursor in the target pathway, ManB and ManC were placed as the upstream module responsible for converting mannose-1-P into GDP-D-mannose. Gmd and WcaG were placed on the other module for the downstream pathway. More specifically, three plasmids pACYCDuet-1, pCDFDuet-1, and pETDuet-1 with different gene copy numbers (the gene copy numbers were defined as 10,20 , and 40, respectively) were utilized to reconstruct the de novo pathway for GDP-L-fucose production. Thus, via expressing two modules either on high, moderate, or low copy number plasmids, nine recombinant host strains (EWL01-09) were constructed to discover the optimal combination of these two modules (Figure. 2). The product was verified by ESI-MS (Figure. S1).

Shake-flask fermentation results showed that individual overexpression of Gmd and WcaG under different copy number plasmids, resulted in the engineered strains EWL01, EWL04, and EWL07, and remarkably improved the titer of GDP-L-fucose to $4.13,3.4$, and $2.6 \mathrm{mg} / \mathrm{L}$, respectively, after 20 hours of fermentation (Figure. 2). These results indicated that higher expression levels of the downstream module favored more the accumulation of intracellular GDP-L-fucose.

In order to bolster the supplementation of precursor GDP-D-mannose, the upstream pathway was considered, generating the recombinant strains EWL02, EWL03, EWL05, EWL06, EWL08, and EWL09. Interestingly, overexpression of the upstream module under low gene dosage (pACYC-manC-manB) led to higher GDP-L-fucose production compared with that under moderate or high gene dosage (pCDF-manC- 
manB or pET-manC-manB). For example, the strains EWL03, EWL06, and EWL09 had 162\%, 65\%, and 58\% higher GDP-L-fucose production compared with the strains EWL02, EWL05, and EWL08, respectively. More specifically, the strain EWL03 (harboring plasmids pET-gmd-wcaG and pACYC-manC-manB) gave the highest GDP-L-fucose titer, which reached $5.33 \mathrm{mg} / \mathrm{L}$.

However, the strain EWL08 (harboring plasmids pACYC-gmd-wcaG and pET-manC-manB) accumulated the lowest GDP-L-fucose concentration $(0.6 \mathrm{mg} / \mathrm{L})$, indicating that higher expression levels of the upstream module severely inhibited the conversion of mannose-6-P to GDP-L-fucose. Indeed, the catalytic efficiency of ManC was 10-fold higher than that of Gmd, consequently resulting in the accumulation of intermediate metabolites (GDP-D-mannose) and a toxic byproduct (pyrophosphate, PPi), which may adversely influence cell growth and product accumulation. ${ }^{13}$

Collectively, these results indicated that relatively higher gene dosage of the downstream pathway and lower gene dosage of the upstream pathway resulted in more GDP-L-fucose yield, potential because this combination alleviated the accumulation of intermediate products and facilitated the efficient transformation of GDP-D-mannose into GDP-L-fucose.

Tuning translation initiation rate through RBS engineering to enhance GDP-L-fucose production

Ribosome binding sites (RBSs) engineering is one of the most widely employed strategies for combinatorial fine-tuning of pathways based on diversification of gene translation levels. To further determine the optimal combination of expression levels in the de novo pathway for GDP-L-fucose biosynthesis, RBS engineering was used as a feasible procedure, which has been widely implemented in many other studies [24-27]. Thus, we adjusted the translation initiation rate of both upstream and downstream modules to further balance the supplementation and consumption of GDP-D-mannose in the target metabolic system.

Subsequently, the standard RBS (RBS-32) [24], the wild type RBS (RBS-WT) of target genes, and the original RBS (RBS-ori) on Duet-1 plasmids were chosen to fine-tune gene expression at the translational level for further improvement of the productivity of GDP-L-fucose (Figure. 3A). In order to define the expression strength of the engineered RBS variants, SDS-PAGE analysis was performed, which rationally characterized the translational activities of the involved RBSs (Figure. S2). The translation strength of the wild type RBSs was distinctly more powerful than that of RBS-ori, while RBS-ori showed higher RBS activity than RBS-32. Each of the module genes was installed under the control of a relatively strong (RBS-WT, represented as 1), moderate (RBS-ori, represented as 2), and weak (RBS-32, represented as 3) RBS. These RBS sequences were paired to generate nine engineered strains to identify the relatively optimal combination (Figure. 3B).

When the Gmd-WcaG module was expressed under the control of relatively weak RBS (RBS-32), the titers of GDP-L-fucose typically decreased, as observed in the strains EWL13, EWL23, and EWL33, which resulted in 2.13, 1.17, and $2.1 \mathrm{mg} / \mathrm{L}$ intracellular GDP-L-fucose concentration, respectively (Figure. 3C). 
These three strains gained a comparatively lower GDP-L-fucose production, probably owing to the accumulation of intermediate metabolites caused by deficient expression of the downstream module.

The strains EWL32 (harboring plasmids pACYC-[RBS-32]-C-[RBS-32]-B and pET-gmd-wcaG) and EWL31 (harboring plasmids pACYC-[RBS-32]-C-[RBS-32]-B and pET-[RBS-WT]-G-[RBS-WT]-W) produced $5.67 \mathrm{mg} / \mathrm{L}$ and $6.77 \mathrm{mg} / \mathrm{L} \mathrm{GDP}$-L-fucose at $20 \mathrm{~h}$, which corresponds to a $6.4 \%$ and $27 \%$ increase, respectively, compare to strain EWL03 (5.33 mg/L). In addition, intracellular GDP-L-fucose accumulated to 3.87, 5.16, and $4.9 \mathrm{mg} / \mathrm{L}$ in EWL12, EWL21, and EWL11, respectively (Figure. 3C). All these titers were slightly reduced compared with the strain EWL31, indicating that relatively excessive protein expression levels of the upstream module blocked the carbon flux to the desired product.

By expressing the upstream pathway on low RBS activity (RBS-32) and expressing the downstream pathway on high RBS activity (RBS-WT), the levels of GDP-L-fucose produced through the de novo synthesis pathway were more elegantly modulated and achieved an optimal GDP-L-fucose production of $6.77 \mathrm{mg} / \mathrm{L}$ in the EWL31 strain.

Effects of inactivating wcaJ on GDP-L-fucose production

Apparently, a common pathway engineering approach to enhance the yield of valuable products is to overexpress the rate-limiting enzymes and inactivate competing pathways to orient the metabolic flux to the pathway of interest. GDP-L-fucose is one of the crucial intermediates for the synthesis of colanic acid [28]. In order to augment the intracellular concentration of GDP-L-fucose, the metabolic flux from GDP-Lfucose to colanic acid was blocked via inactivating the gene wcaJ encoding the UDP-glucose lipid carrier transferase [29]. Specifically, the CRISPR/Cas9 system was implemented to rapidly and efficiently knock out wcaJ in E. coli BL21 (DE3) [30-31].

The shake-flask fermentation showed that the intracellular GDP-L-fucose concentration reached $12.26 \mathrm{mg} / \mathrm{L}(2.75 \mathrm{mg} / \mathrm{g} \mathrm{DCW})$ at $20 \mathrm{~h}$ in EWL34 and gained a 1.53-fold improvement compared with that in EWL31. Furthermore, dry cell weight for strain EWL34 was 4.45, illustrating 7\% less growth compared to EWL31 (4.79 at $20 \mathrm{~h}$ ). Therefore, the deletion of wcaJ effectively bolstered GDP-L-fucose titers, and the cell biomass and growth conditions of the engineered strain were not apparently affected (Figure. 4). This result indicated that knocking out wcaJ blocked the flux from GDP-L-fucose to colonic acid and significantly improved the accumulation of the target product.

Effect of facilitating the cofactor NADPH regeneration pathway and GTP biosynthesis pathway on GDPL-fucose production

Cofactors are involved in large numbers of intracellular reactions and critically manipulate carbon metabolism and redox balance. As the most essential redox carriers corresponding to cellular metabolism, $\mathrm{NAD}(\mathrm{P})^{+}, \mathrm{NAD}(\mathrm{P}) \mathrm{H}$ and nucleotides play pivotal roles in the efficient production of biochemicals and pharmaceuticals [32-33]. Coupling cofactor engineering with metabolic flux engineering can increase the efficiency and productivity of large-scale processes for products of interest. 
For this reason, we regenerated the cofactor systems by strengthening the endogenous cofactor pathway related to the desired metabolite.

Previous studies illustrated that overexpression of zwf gene for NADPH regeneration [16] or gsk gene for GTP regeneration [17] was able to optimally enhance the intracellular GDP-L-fucose accumulation than up-regulating other genes in pentose phosphate pathway or the guanosine nucleotides biosynthetic pathway. To further boost the concentration of intracellular GDP-L-fucose, both glucose-6-phosphate dehydrogenase (Zwf) and guanosine-inosine kinase (Gsk) were overexpressed to improve the balance of consumption and regeneration of related cofactors. Figure. 5 illustrates that introduction of cofactor engineering module significantly improved the production of intracellular GDP-L-fucose.

The recombinant E. coli overexpressing Zwf (EWL35) showed $15 \mathrm{mg} / \mathrm{L}(3.6 \mathrm{mg} / \mathrm{g} \mathrm{DCW})$ intracellular GDP-L-fucose accumulation, and thus the titer in this strain was increased by $22.4 \%$ compared with that in the EWL34 strain. Furthermore, an even better performance was achieved in the Gsk-overexpressing strain EWL36 (with an increase of 31.7\% compared with EWL34), indicating that strengthening the GTP biosynthesis pathway by overexpression of Gsk, which catalyzes the bioconversion of inosine and guanosine to IMP and GMP, respectively [34], efficiently enhanced GDP-L-fucose production. It is worth noting that the cell growth of strain EWL36 was noticeably boosted by overexpression of Gsk. This might be attributed to the reinforcement of biosynthesis of guanosine nucleotide, an indispensable precursor for the synthesis of nutrient elements such as vitamins and amino acids $[25,35]$.

Given that ManC demands GTP for GDP-D-mannose biotransformation and that WcaG needs NADPH for the biosynthesis of target products, efficient feeding of these two cofactors is critical for improving production. Thus, we simultaneously overexpressed Zwf and Gsk in strains harboring plasmids for GDPL-fucose production, generating the strain EWL37. As expected, EWL37 was also amenable to efficiently produce GDP-L-fucose and the accumulation of intracellular GDP-L-fucose reached to $18.33 \mathrm{mg} / \mathrm{L}$ $(4.5 \mathrm{mg} / \mathrm{g} \mathrm{DCW})$, corresponding to a $49.5 \%$ increase compared to that in EWL34. These results suggested that the proper cofactor engineering made the production of GDP-L-fucose more efficient.

Production of GDP-L-fucose in 3-L bioreactors

To obtain high titers of GDP-L-fucose under certain conditions for the engineered strain, fed-batch cultivation of the optimal GDP-L-fucose accumulating strain EWL37 was employed. The fermentation results illustrated that cellular growth was continuously increasing till the end of the fermentation. When the initial glucose was exhausted, glucose feeding started and was limited at a constant flow rate of $8 \mathrm{~g}$ glucose per hour to control the production of acetic acid, which could be toxic to cell growth. After the addition of IPTG (the final concentration was $0.1 \mathrm{mM}$ ) at $16 \mathrm{~h}$, the product started to accumulate slowly within the first 8 hours. Then, GDP-L-fucose rapidly accumulated with sustained consumption of glucose and oxygen. After $24 \mathrm{~h}$ from induction, the production of GDP-L-fucose could be accumulated to

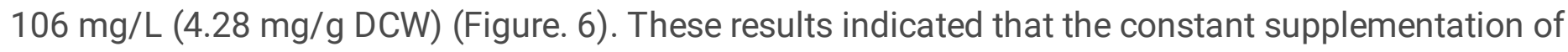
carbon source, efficient NADPH and GTP regeneration and metabolic flux toward the desired biosynthesis pathway noticeably boosted the biosynthesis of GDP-L-fucose. Although the titer is not optimal 
compared with others, the specific GDP-L-fucose content is demonstrably superior to previous $3.5 \mathrm{mg} / \mathrm{g}$ DCW, which proved that more efficient cell factories are established in our research.

\section{Conclusion}

In this study, combinatorial modular pathway engineering strategy was carried out to systematically and synergistically regulate the cellular metabolic networks for the biosynthesis of GDP-L-fucose in E. coli. A noticeable improvement in GDP-L-fucose production was achieved by fine-tuning the key enzymes in the metabolic pathway, deleting the bypass gene, and strengthening cofactors regeneration. It is worth noting that the allosteric inhibition of GDP-L-fucose on the enzyme GDP-D-mannose-4,6-dehydratase (Gmd) of the de novo pathway exerts negative effects on intracellular product accumulation and may be a further obstacle in reaching higher intracellular yields [36]. Hence, the modification of target enzyme and/or the export of GDP-L-fucose from recombinant E. coli strains by heterogeneous expression of GDP-L-fucose transporter can be crucial and feasible strategies [37]. In addition, for further production of GDP-L-fucose, more efforts should be directed in the analysis of the global metabolic flow and the optimization of the fermentation conditions.

\section{Materials And Methods}

Microorganisms, plasmids, medium and cultivation conditions

All strains and plasmids used in this study are listed in Tables S1 and S2, respectively. E. coli DH5a was used for plasmid construction and propagation and E. coli BL21(DE3) was used as the initial host strain for GDP-L-fucose production. The vectors pETDuet-1, pCDFDuet-1, and PACYCDuet-1 were employed for cloning and subcloning. All restriction enzymes, Primer Star DNA polymerase and T4 DNA ligase were purchased from Takara (Kyoto, Japan). Molecular kits utilized for gel and plasmid extraction and DNA purification were purchased from TIANGEN (Beijing, China).

The engineered strains were grown in Luria-Bertani (LB) medium ( $10 \mathrm{~g} / \mathrm{L}$ tryptone, $5 \mathrm{~g} / \mathrm{L}$ yeast extract, and $10 \mathrm{~g} / \mathrm{L} \mathrm{NaCl}$ ) or on Luria-Bertani agar plates $(15 \mathrm{~g} / \mathrm{L}$ agar). The glucose-defined medium was employed for shake flask cultivation and 3- $\mathrm{L}$ fed-batch bioreactor fermentation. The glucose-defined medium contained: $20 \mathrm{~g} / \mathrm{L}$ glucose, $13.5 \mathrm{~g} / \mathrm{L} \mathrm{KH}_{2} \mathrm{PO}_{4}, 4.0 \mathrm{~g} / \mathrm{L}\left(\mathrm{NH}_{4}\right)_{2} \mathrm{HPO}_{4}, 1.7 \mathrm{~g} / \mathrm{L}$ citric acid, $1.4 \mathrm{~g} / \mathrm{L}$ $\mathrm{MgSO}_{4} \cdot 7 \mathrm{H}_{2} \mathrm{O}, 4.5 \mathrm{mg} / \mathrm{L}$ thiamine, and $10 \mathrm{ml} / \mathrm{L}$ trace element solution. The trace element solution contained: $10 \mathrm{~g} / \mathrm{L} \mathrm{FeSO}_{4} \cdot 7 \mathrm{H}_{2} \mathrm{O}, 2.2 \mathrm{~g} / \mathrm{L} \mathrm{ZnSO}_{4} \cdot 7 \mathrm{H}_{2} \mathrm{O}, 1.0 \mathrm{~g} / \mathrm{L} \mathrm{CuSO}_{4} \cdot 5 \mathrm{H}_{2} \mathrm{O}, 0.38 \mathrm{~g} / \mathrm{L} \mathrm{MnSO}_{4} \cdot \mathrm{H}_{2} \mathrm{O}, 0.02 \mathrm{~g} / \mathrm{L}$ $\mathrm{Na}_{2} \mathrm{~B}_{4} \mathrm{O}_{7} \cdot 10 \mathrm{H}_{2} \mathrm{O}, 0.1 \mathrm{~g} / \mathrm{L}\left(\mathrm{NH}_{4}\right)_{6} \mathrm{Mo}_{7} \mathrm{O}_{24}, 2.0 \mathrm{~g} / \mathrm{L} \mathrm{CaCl}_{2}$, and the $\mathrm{pH}$ was regulated to 6.8 . The feeding solution used in the fed-batch fermentation contained: $800 \mathrm{~g} / \mathrm{L}$ glucose, $20 \mathrm{~g} / \mathrm{L} \mathrm{MgSO}{ }_{4} \cdot 7 \mathrm{H}_{2} \mathrm{O}$, and $0.2 \mathrm{~g} / \mathrm{L}$ thiamine.

Construction of plasmids and substitution of ribosome binding sites

Plasmid construction and DNA manipulation were performed following standard molecular cloning protocols. Primers used in this study are listed in Table S3. Genes manB, manC, gmd, wcaG, gsk, and zwf 
were amplified from the E. coli MG1655 chromosome using primer pairs Ndel-manB-F/R, Ncol-manC-F/R, Ncol-gmd-F/R, Ndel-wcaG-F/R, Ncol-gsk-F/R, and Ndel-zwf-F/R, respectively. First, Ncol digested PCR products were ligated to Ncol digested pETDuet-1, pCDFDuet-1, and pACYCDuet-1 to construct the plasmids pET-manC, pCDF-manC, pACYC-manC, pET-gmd, pCDF-gmd, pACYC-gmd, and pCDF-gsk. Then, Ndel digested PCR products were ligated to the Ndel digested plasmids mentioned before to construct pET-manC-manB, pCDF-manC-manB, pACYC-manC-manB, pET-gmd-wcaG, pCDF-gmd-wcaG, pACYC-gmdwcaG, and pCDF-gsk-zwf.

The ribosome binding site (RBS)-32, which served as a standard ribosome binding element, was chosen from a previous study [24]. The original RBS in the plasmids mentioned before was defined as RBS-ori, and RBS-ori was substituted by RBS-32, and RBS-WT (the wild type RBS of each gene in E. coli BL21 (DE3) genome). For constructing pACYC-[RBS-i]-C-[RBS-i]-B and pET-[RBS-i]-G-[RBS-i]-W ( $i=32, W T)$, the fragments and vectors with different RBS were amplified from pACYC-manC-manB and $p E T$-gmd-wcaG with primers manC-[RBS-i]-F/R, manB-[RBS-i]-F/R, gmd-[RBS-i]-F/R, and wcaG-[RBS-i]-F/R. Subsequently, the fragments and vectors were cloned using One Step Cloning Kit (Vazyme Biotech Co., Ltd., Nanjing, China) to construct plasmids pACYC-[RBS-32]-C-[RBS-32]-B, pACYC-[RBS-WT]-C-[RBS-WT]-B, pET-[RBS-32]G-[RBS-32]-W, and pET-[RBS-WT]-G-[RBS-WT]-W.

Gene inactivation through CRISPR-Cas9

A two-plasmid-based CRISPR-Cas9 system for E. coli genome editing was utilized to delete the gene wcaJ. The plasmid pCas contains the cas9 gene and the single-guide RNA (sgRNA) for the deletion of plasmid pTargetF. The pTargetF harbors sgRNA with certain N20 sequence targeting the gene needed to be deleted. The pTargetF and the template DNA were electroporated into cells with $\mathrm{pCas}$, and $10 \mathrm{mM}$ arabinose was added for $\lambda$-Red induction to promote homologous recombination. After that, Cas 9 was used to cut the pTargetF via adding $0.5 \mathrm{mM}$ Isopropyl- $\beta$-D-thiogalactopyranoside (IPTG) to induce the expression of sgRNA-pMB1 and pCas was cured by overnight cultivation of the colonies at $42{ }^{\circ} \mathrm{C}[30]$.

SDS-PAGE analysis for gene expression levels assay

Sodium dodecyl sulfate-polyacrylamide gel electrophoresis (SDS-PAGE) was applied to compare the expression levels of overexpressed enzymes involved in the de novo pathway for GDP-fucose biosynthesis. Culture samples $(2 \mathrm{~mL})$ were taken $6 \mathrm{~h}$ after induction and the cells were harvested by centrifugation at $5,000 \times \mathrm{g}$. Cell pellets were resuspended in $50 \mathrm{mM}$ Tris- $\mathrm{HCl}(\mathrm{pH} 7.5)$ and loading buffer was added before disrupting by boiling for $10 \mathrm{~min}$. After that, the supernatant was collected via centrifugation at $10,000 \times \mathrm{g}$ for $10 \mathrm{~min}$. Samples of the supernatant of different host strains were subjected to SDS-PAGE analysis [7].

Gels were electrophoresed at $80 \mathrm{~V}$ until the samples fully passed through the concentrate. Then, the voltage was maintained at $120 \mathrm{~V}$. After that, the gel was stained by Coomassie brilliant blue (Biotechnology company, Shanghai, China) for $3 \mathrm{~h}$. Products were visualized using an automatic imageanalysis system (Tanon Science \& Technology Co., Ltd., Shanghai, China) [38]. 
Optical density was used to determined the dry cell weight (DCW) and the conversion equation is $10 D_{600}$ $=0.35 \mathrm{~g} / \mathrm{L}$. the samples were diluted to the optical density between 0.1 and 0.8 before detecting. Commercialized SBA-40C biosensor analyzer (Biology Institute of Shandong Academy of Sciences) was used to measure the concentration of glucose.

For shake-flask cultivation, strains were inoculated into $4 \mathrm{~mL}$ of LB medium and grown overnight at $200 \mathrm{rpm}$ and $37^{\circ} \mathrm{C}$. Then, $0.5 \mathrm{~mL}$ of the culture were inoculated into $25 \mathrm{~mL}$ of defined medium containing relevant antibiotics in 250-mL shake flasks at $200 \mathrm{rpm}$ and $37^{\circ} \mathrm{C}$. IPTG was added to a final concentration of $0.1 \mathrm{mM}$ when cells had reached an optical density $\left(\mathrm{OD}_{600}\right)$ between 0.6 and 0.8 . Then, the cultivation was performed at $25^{\circ} \mathrm{C}$. Antibiotics were added at certain final concentrations.

\section{Fed-batch fermentation in a 3-L bioreactor}

Fed-batch cultivation was employed in a 3-L bioreactor containing $1.0 \mathrm{~L}$ defined medium. After the complete depletion of $20 \mathrm{~g} / \mathrm{L}$ glucose added initially, feeding solution was fed at a constant feed rate of $10 \mathrm{~mL} / \mathrm{h}$. When $\mathrm{OD}_{600}$ reached $15,0.1 \mathrm{mM}$ IPTG was added to induce the expression of target genes. The $\mathrm{pH}$ was maintained at 6.8 via the addition of $25 \%$ aqueous ammonia. The temperature was maintained at $25{ }^{\circ} \mathrm{C}$ and the inoculation density was $5 \%$. The air flow and agitation speed were $1.5 \mathrm{vvm}$ and $700 \mathrm{rpm}$, respectively.

Identification and measurement of intracellular GDP-L-fucose concentration

To identify the biosynthesis of GDP-L-fucose, the GDP-L-fucose standard and crude product samples were analyzed by electro-spray ionization-mass spectroscopy (ESI-MS) on a Waters MALDI SYNAPT QTOF MS (Milford, MA, USA) in negative ion mode, under standard conditions.

For the measurement of intracellular GDP-L-fucose, $5 \mathrm{~mL}$ of the culture were harvested by centrifugation $(5,000 \times \mathrm{g}, 15 \mathrm{~min})$. Cells resuspended in extraction buffer were disrupted via sonication. After centrifugation $(10,000 \times \mathrm{g}, 10 \mathrm{~min})$, the supernatant was analyzed by a high-performance liquid chromatography (HPLC) system (Agilent Technologies, USA) with an Inertsil ODS-SP column (GL Sciences, Kyoto, Japan) and detected by UV-photometry at a wavelength of $254 \mathrm{~nm}$. The column was eluted at a flow rate of $0.6 \mathrm{~mL} / \mathrm{min}$ sequentially by certain procedure [19].

\section{Supplementary Information}

Table S1. Strains used in this study. Table S2. Plasmids used in this study. Table S3. Primers used in this study. Fig. S1. Analysis of GDP-L-fucose by ESI-MS. (a) GDP-L-fucose standard; (b) GDP-L-fucose from fermentation broth. Fig. S2. SDS-PAGE analysis of soluble fraction of different engineering strain.

\section{Abbreviations}


GDP-L-fucose:guanosine 5'-diphosphate-L-fucose; WcaJ:UDP-glucose lipid carrier transferase; DCW:dry cell weight; HMOs:Human milk oligosaccharides; ManA:mannose-6-phosphate isomerase; ManB:phosphomannomutase; ManC:a-D-mannose-1-phosphate guanyltransferase; Gmd:GDP-Dmannose-4,6-dehydratase; WcaG:GDP-L-fucose synthetase; GTP:guanosine 5'-triphosphate; Fkp:fucokinase/fucose-L-phosphate guanylyltransferase; PPP:pentose phosphate pathway; RBS:ribosome binding site; Zwf:glucose-6-phosphate dehydrogenase; Gsk:guanosine-inosine kinase; IPTG:isopropyl- $\beta$-D-thiogalactopyranoside; $\mathrm{OD}_{600}$ :optical density at the wavelength of $600 \mathrm{~nm}$.

\section{Declarations}

\section{Authors' contributions}

LW and YYZ designed the study. LW performed the experiments, analyzed the experiment data and drafted the manuscript. WLZ, YYZ, WL, and WMM commented on the manuscript. All authors read and approved the final manuscript.

\section{Acknowledgements}

Not applicable.

\section{Funding}

This work was supported by the National Natural Science Foundation of China (No.31922073, 31801583), and the National Science Foundation of Jiangsu Province (No. BK20180607).

\section{Availability of data and materials}

The data supporting the results of the article are included in this manuscript and supplementary information.

\section{Ethics approval and consent to participate}

Not applicable.

\section{Consent for publication}

Not applicable.

\section{Competing interests}

The authors declare that they have no competing interests.

\section{Author details}


${ }^{1}$ State Key Laboratory of Food Science and Technology, Jiangnan University, Wuxi, Jiangsu, 214122, China. ${ }^{2}$ International Joint Laboratory on Food Safety, Jiangnan University, Wuxi 214122, China.

\section{References}

1. Kong F. Recent studies on reaction pathways and applications of sugar orthoesters in synthesis of oligosaccharides. Carbohyd Res. 2007;342:345-373.

2. Koizumi S. Large-scale production of oligosaccharides using bacterial functions. Trends Glycosci Glyc. 2003;15:65-74.

3. Bode L. Human milk oligosaccharides: Every baby needs a sugar mama. Glycobiology. 2012;22:1147-1162.

4. Bych K, Miks MH, Johanson T, Hederos MJ, Vigsnaes LK, Becker P. Production of HMOs using microbial hosts - from cell engineering to large scale production. Curr Opin Biotechnol. 2019;56:130137.

5. Faijes M, Castejon-Vilatersana M, Val-Cid C, Planas A. Enzymatic and cell factory approaches to the production of human milk oligosaccharides. Biotechnol Adv. 2019;37:667-697.

6. Petschacher B, Nidetzky B. Biotechnological production of fucosylated human milk oligosaccharides: Prokaryotic fucosyltransferases and their use in biocatalytic cascades or whole cell conversion systems. J Biotechnol. 2016;235:61-83.

7. Zhai Y, Han D, Pan Y, Wang S, Fang J, Wang P, Liu X. Enhancing GDP-fucose production in recombinant Escherichia coli by metabolic pathway engineering. Enzyme Microb Technol. 2015;69:38-45.

8. Coyne MJ, Reinap B, Lee MM, Comstock LE. Human symbionts use a host-like pathway for surface fucosylation. Science. 2005;307:1778-1781.

9. Yi W, Liu X, Li Y, Li J, Xia C, Zhou G, Zhang W, Zhao W, Chen X, Wang PG. Remodeling bacterial polysaccharides by metabolic pathway engineering. Prco Natl Acad Sci U S A. 2009;106:4207-4212.

10. Liu TW, Ito H, Chiba Y, Kubota T, Sato T, Narimatsu H. Functional expression of Lfucokinase/guanosine 5'-diphosphate-L-fucose pyrophosphorylase from Bacteroides fragilis in Saccharomyces cerevisiae for the production of nucleotide sugars from exogenous monosaccharides. Glycobiology. 2011;21:1228-1236.

11. Mattila P, Rabina J, Hortling S, Helin J, Renkonen R. Functional expression of Escherichia coli enzymes synthesizing GDP-L-fucose from inherent GDP-D-mannose in Saccharomyces cerevisiae. Glycobiology. 2000;10:1041-1047.

12. Nakayama K, Maeda Y, Jigami Y. Interaction of GDP-4-keto-6-deoxymannose-3,5-epimerase-4reductase with GDP-mannose-4,6-dehydratase stabilizes the enzyme activity for formation of GDPfucose from GDP-mannose. Glycobiology. 2003;13:673-680.

13. Chin YW, Park JB, Park YC, Kim KH, Seo JH. Metabolic engineering of Corynebacterium glutamicum to produce GDP-L-fucose from glucose and mannose. Bioprocess Biosyst Eng. 2013;36:749-756. 
14. Byun SG, Kim MD, Lee WH, Lee KJ, Han NS, Seo JH. Production of GDP-L-fucose, L-fucose donor for fucosyloligosaccharide synthesis, in recombinant Escherichia coli. Appl Microbiol Biotechnol. 2007;74:768-775.

15. Lee WH, Han NS, Park YC, Seo JH. Modulation of guanosine 5'-diphosphate-D-mannose metabolism in recombinant Escherichia coli for production of guanosine 5'-diphosphate-L-fucose. Bioresour Technol. 2009;100:6143-6148.

16. Lee WH, Chin YW, Han NS, Kim MD, Seo JH. Enhanced production of GDP-L-fucose by overexpression of NADPH regenerator in recombinant Escherichia coli. Appl Microbiol Biotechnol. 2011;91:967-976.

17. Lee WH, Shin SY, Kim MD, Han NS, Seo JH. Modulation of guanosine nucleotides biosynthetic pathways enhanced GDP-L-fucose production in recombinant Escherichia coli. Appl Microbiol Biotechnol. 2012;93:2327-2334.

18. Baumgärtner F, Seitz L, Sprenger GA, Albermann C. Construction of Escherichia coli strains with chromosomally integrated expression cassettes for the synthesis of 2'-fucosyllactose. Microb Cell Fact. 2013;12:40.

19. Huang D, Yang K, Liu J, Xu Y, Wang Y, Wang R, Liu B, Feng L. Metabolic engineering of Escherichia coli for the production of 2'-fucosyllactose and 3-fucosyllactose through modular pathway enhancement. Metab Eng. 2017;41:23-38.

20. Biggs BW, De Paepe B, Santos CNS, De Mey M, Ajikumar PK. Multivariate modular metabolic engineering for pathway and strain optimization. Curr Opin Biotechnol. 2014;29:156-162.

21. Wu G, Yan Q, Jones JA, Tang YJ, Fong SS, Koffas MAG. Metabolic burden: Cornerstones in synthetic biology and metabolic engineering applications. Trends Biotechnol. 2016;34:652-664.

22. Ajikumar PK, Xiao WH, Tyo KEJ, Wang Y, Simeon F, Leonard E, Mucha O, Phon TH, Pfeifer B, Stephanopoulos $\mathrm{G}$. Isoprenoid pathway optimization for taxol precursor overproduction in Escherichia coli. Science. 2010;330:70-74.

23. Jeschek M, Gerngross D, Panke S. Combinatorial pathway optimization for streamlined metabolic engineering. Curr Opin Biotechnol. 2017;47:142-151.

24. Xu P, Gu Q, Wang W, Wong L, Bower AGW, Collins CH, Koffas MAG. Modular optimization of multigene pathways for fatty acids production in E. coli. Nat Commun. 2013;4:1409.

25. Lin Z, Xu Z, Li Y, Wang Z, Chen T, Zhao X. Metabolic engineering of Escherichia coli for the production of riboflavin. Microb Cell Fact. 2014;13:104.

26. Wu J, Cheng S, Cao J, Qao J, Zhao GR. Systematic optimization of limonene production in engineered Escherichia coli. J Agric Food Chem. 2019;67:7087-7097.

27. Zhang J, Weng H, Zhou Z, Du G, Kang Z. Engineering of multiple modular pathways for high-yield production of 5-aminolevulinic acid in Escherichia coli. Bioresour Technol. 2019;274:353-360.

28. Wu H, Chen S, Ji M, Chen Q, Shi J, Sun J. Activation of colanic acid biosynthesis linked to heterologous expression of the polyhydroxybutyrate pathway in Escherichia coli. Int J Biol Macromol. 2019;128:752-760. 
29. Sander JD, Joung JK. CRISPR-Cas systems for editing, regulating and targeting genomes. Nat Biotechnol. 2014;32:347-355.

30. Jiang Y, Chen B, Duan C, Sun B, Yang J, Yang S. Multigene Editing in the Escherichia coli Genome via the CRISPR-Cas9 System. Appl Environ Microbiol. 2015;81:2506-2514.

31. Wang $M$, Chen $B$, Fang $Y$, Tan $T$. Cofactor engineering for more efficient production of chemicals and biofuels. Biotechnol Adv. 2017;35:1032-1039.

32. Chen X, Li S, Liu L. Engineering redox balance through cofactor systerms. Trends Biotechnol. 2014;32:337-343.

33. Dumon C, Priem B, Martin SL, Heyraud A, Bosso C, Samain E. In vivo fucosylation of lacto-Nneotetraose and lacto-N-neohexaose by heterologous expression of Helicobacter pylori alpha-1,3 fucosyltransferase in engineered Escherichia coli. Glycoconj J. 2001;18:465-474.

34. Ledesma-Amaro R, Jimenez A, Santos MA, Revuelta JL. Biotechnological production of feed nucleotides by microbial strain improvement. Process Biochem. 2013;48:1263-1270.

35. Shi T, Wang Y, Wang Z, Wang G, Liu D, Fu J, Chen T, Zhao X. Deregulation of purine pathway in Bacillus subtilis and its use in riboflavin biosynthesis. Microb Cell Fact. 2014;13:101.

36. Somoza JR, Menon S, Schmidt H, Joseph-Mccarthy D, Dessen A, StahI ML, Somers WS, Sullivan FX. Structural and kinetic analysis of Escherichia coli GDP-mannose 4,6-dehydratase provides insights into the enzyme's catalytic mechanism and regulation by GDP-fucose. Structure. 2000;8:123-135.

37. Forster-Fromme K, Schneider S, Sprenger GA, Albermann C. Functional expression of a human GDPL-fucose transporter in Escherichia coli. Biotechnol Lett. 2017;39:219-226.

38. Zhao M, Huang D, Zhang X, Koffas MAG, Zhou J, Deng Y. Metabolic engineering of Escherichia coli for producing adipic acid through the reverse adipate-degradation pathway. Metab Eng. 2018;47:254262.

\section{Figures}




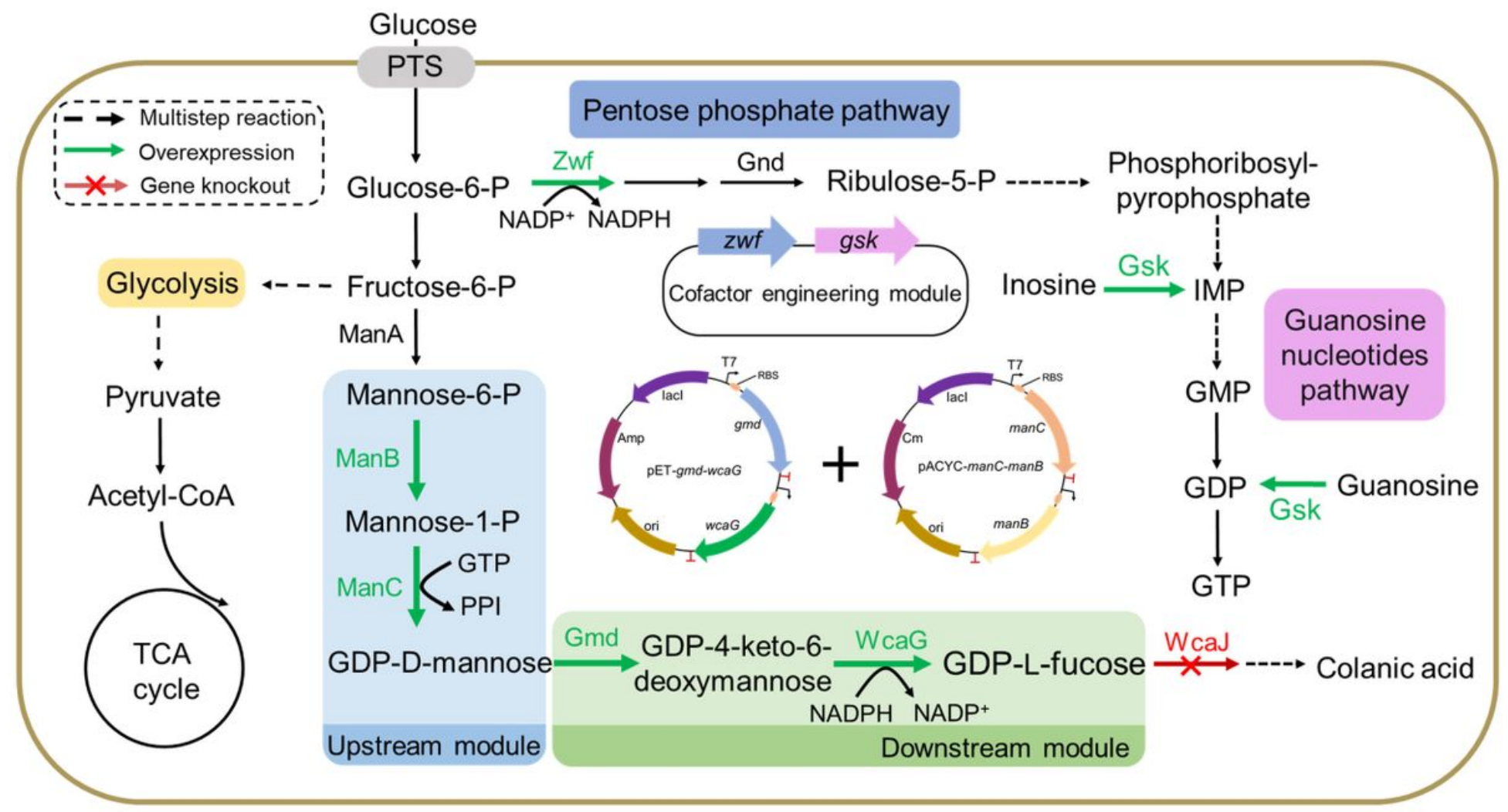

Figure 1

Combinatorial modular pathway engineering strategy for the production of GDP-L-fucose in E. coli. Upstream module contains ManB and ManC. Downstream module contains Gmd and WcaG. Cofactor engineering module contains Zwf and Gsk. Green arrows represent overexpressed enzymes and red arrow represents the inactivated enzyme. ManA, mannose 6-phosphate isomerase; ManB, phosphomannomutase; ManC, a-D-mannose-1-phosphate guanylyltransferase; Gmd, GDP-D-mannose4,6-dehydratase; WcaG, GDP-L-fucose synthetase; Zwf, glucose-6-phosphate dehydrogenase; Gnd, 6phosphogluconate dehydrogenase; WcaJ, UDP-glucose lipid carrier transferase; Gsk, guanosine-inosine kinase. 


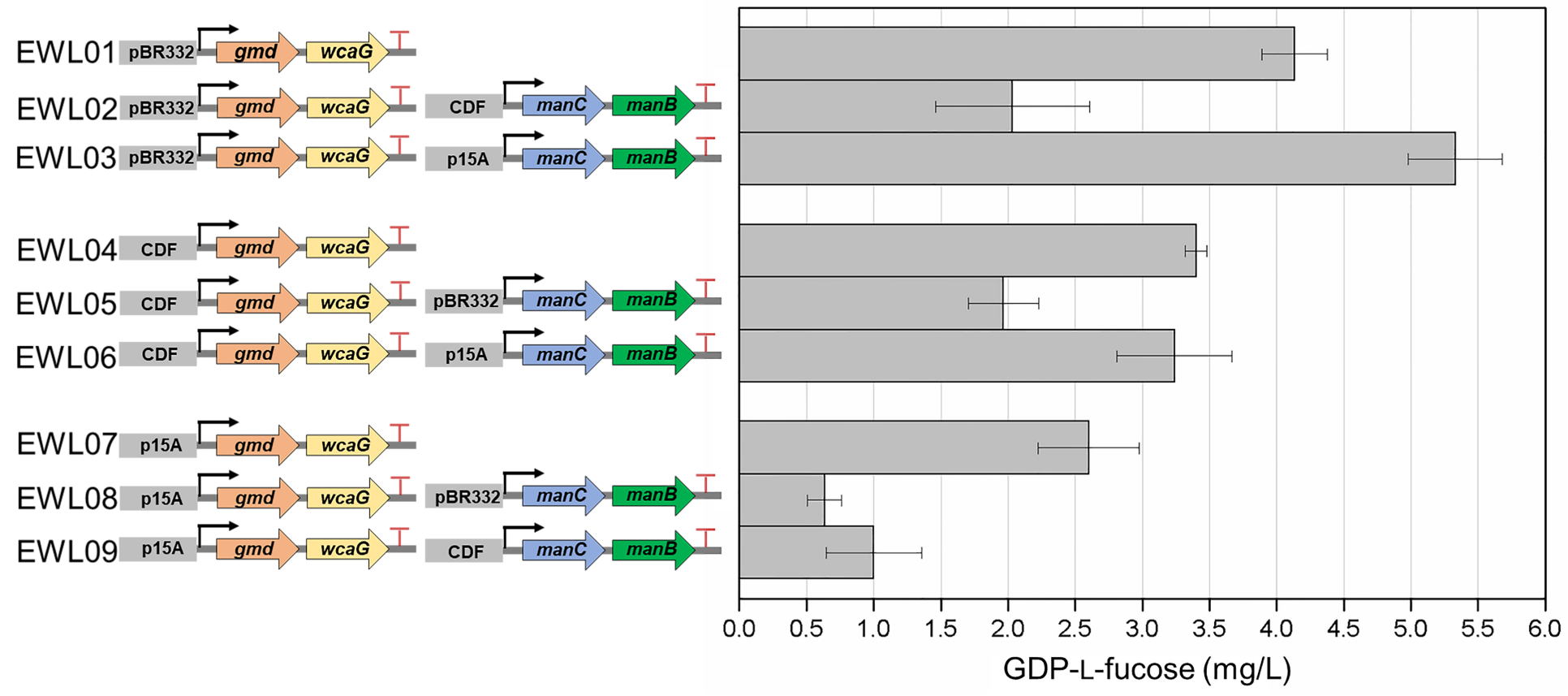

Figure 2

Combinations of enzymes in the de novo GDP-L-fucose synthesis pathway by varying gene copy number. The origins of pETDuet-1, pCDFDuet-1, and pACYCDuet-1 are pBR322, CDF, and p15A, respectively.

A RBS-32: GTCACACAGGAAAGTACCAT TATA CCATGGGTATG (NCOl) GTCACACAGGAAAGTACCAT CATATG (Ndel) RBS-ori : AAGAAGGAGA TATA CCATGGGTATG (Ncol) RBS-WT: AAGAAGGAGA CATATG (Ndel)

ACGCGAACGCGTTGAAACTGAATAAATTCAAAAATACAGAGGAATAATAC TATACCATGGGTATG ( $\mathrm{gmd}$ ) TGCTGAAATCTCACGGCTACGACGTGGCGATCGCGCTGGAGTCATAAATG CATATG (wcaG) GATAAAGAGAACGTGTTACGTCAATTTATAAATGATATTCGGGGATAATT CCATGGGTATG ( $m a n C$ ) TTCGGTCAGGGCCAACTATTGCCTGAAAAAGGGTAACGAT CATATG (manB)

B
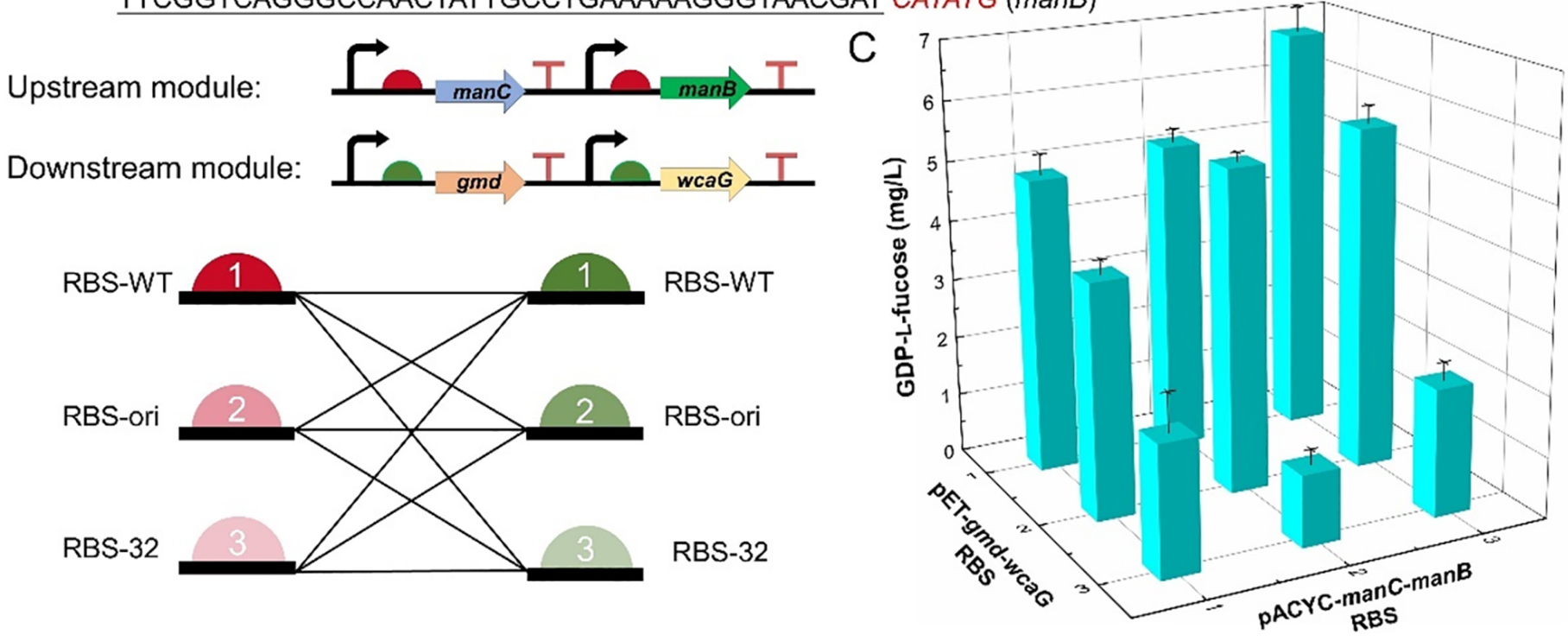

Figure 3 
Improving GDP-L-fucose production via tuning translation initiation rate. (A) RBSs sequence involved in this study: RBS core sequences are underlined; Italic red sequence shows restriction sites. (B) Schematic design of different RBS combinations. (C) Optimization of GDP-L-fucose production by regulating the RBS strength of upstream and downstream modules in strain EWL03. These two modules were expressed either under the control of a relatively strong (RBS-WT, represented as 1), moderate (RBS-ori, represented as 2), or weak (RBS-32, represented as 3) RBS.

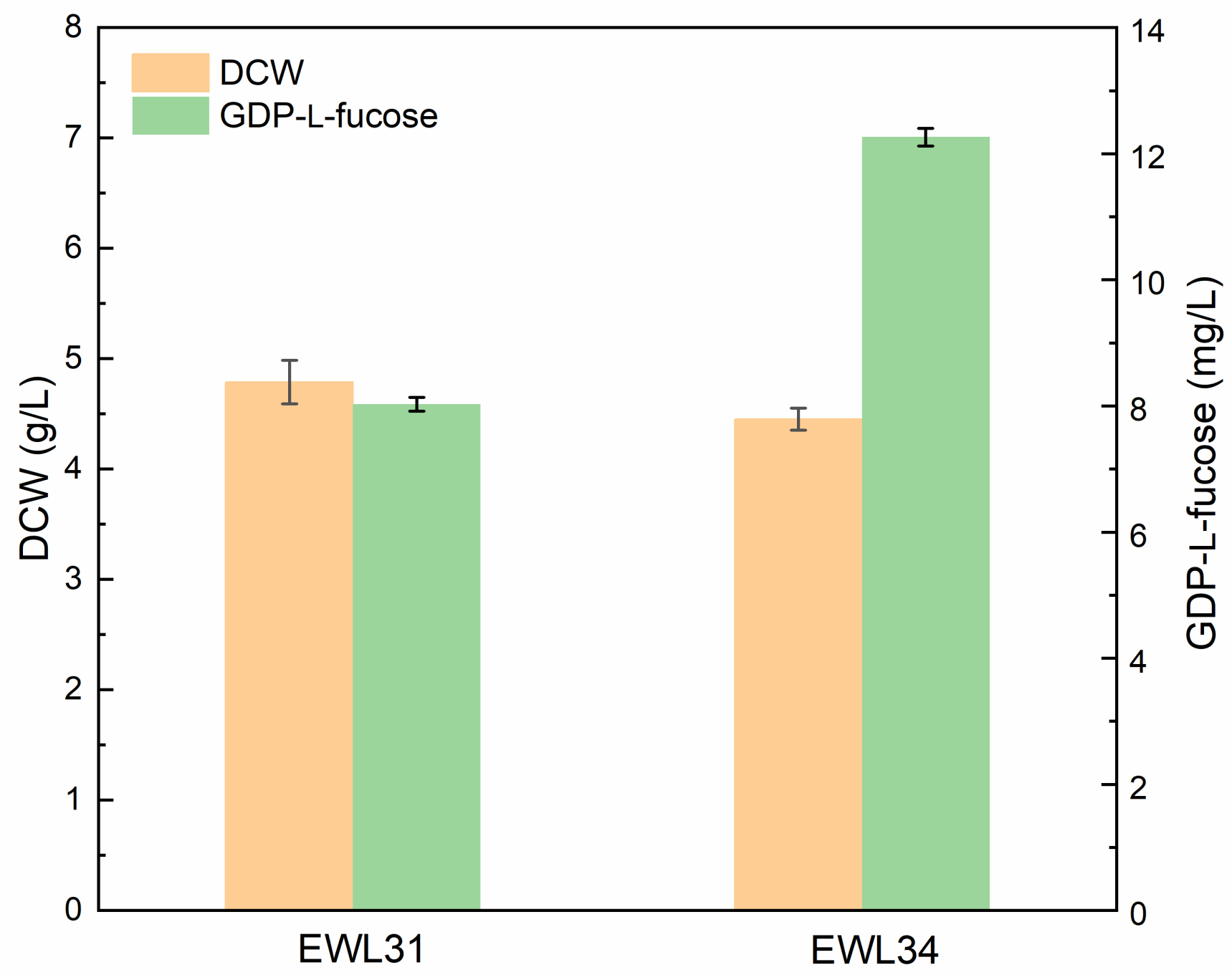

\section{Figure 4}

Fermentation results of strains EWL31 and EWL32. The gene wcaJ was deleted in strain EWL32. 


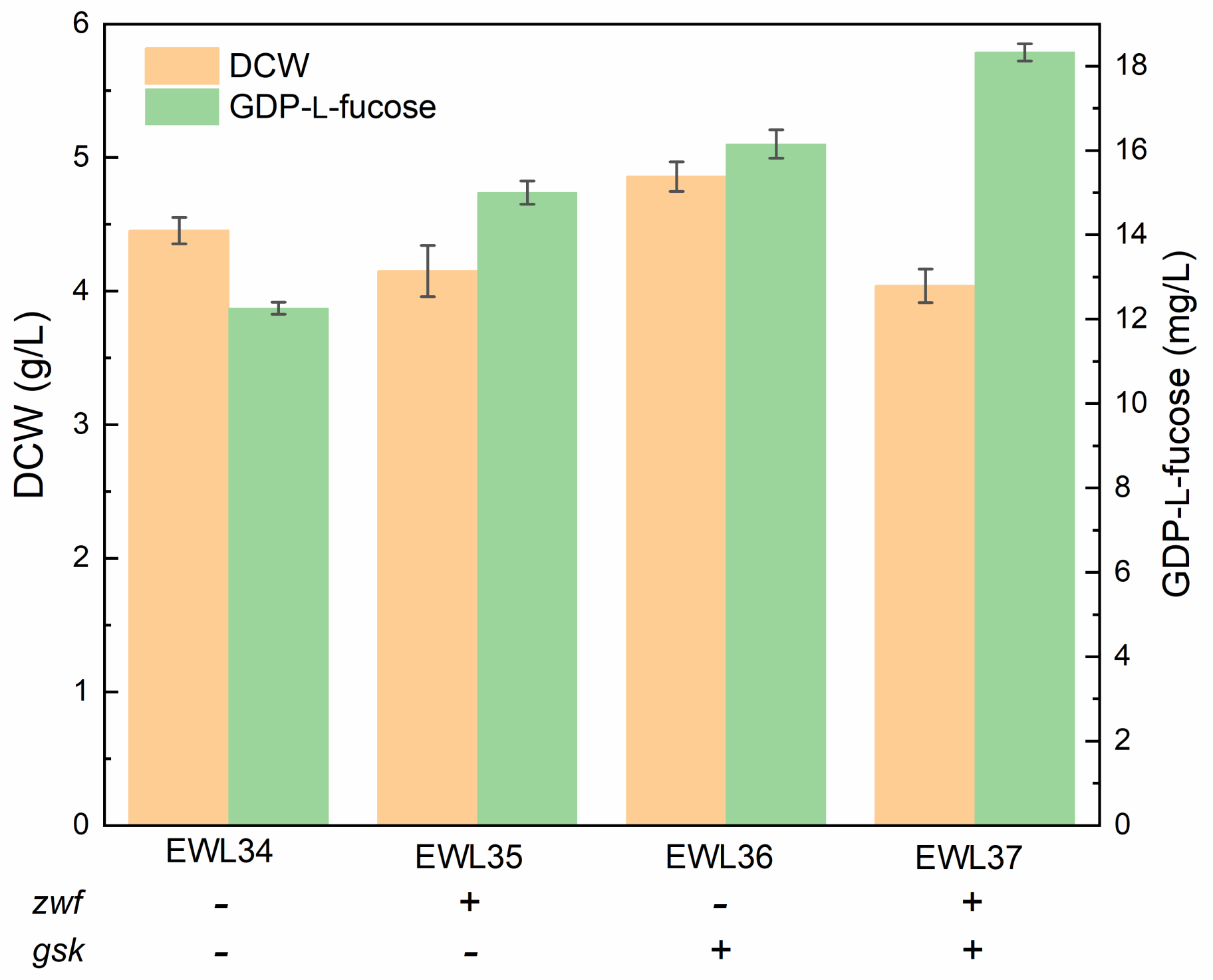

Figure 5

Shaken flask culture results for different engineered strains that considered cofactor regeneration. 


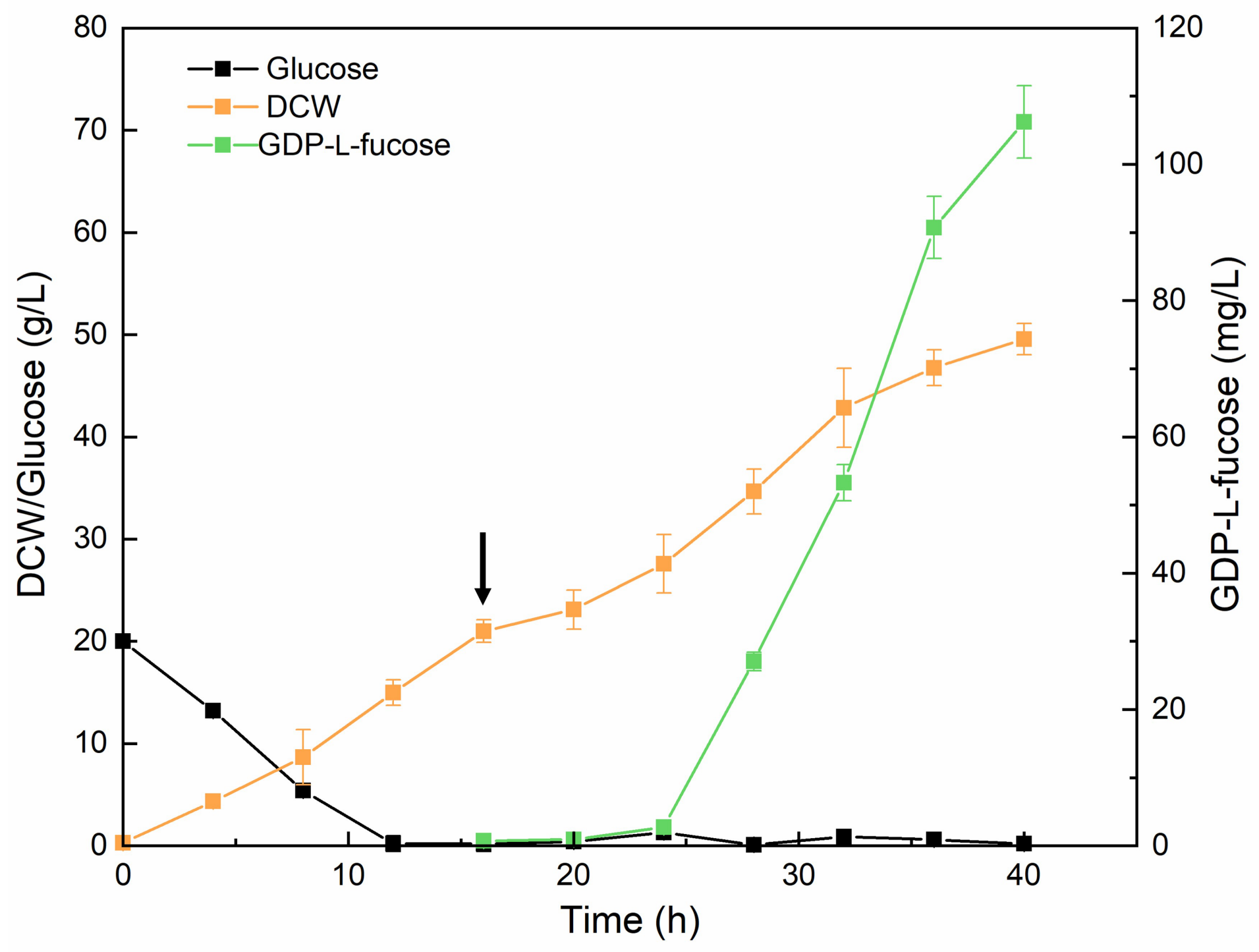

Figure 6

Fed-batch cultivation associated with the optimal strain EWL37. Arrow indicates the addition of $0.1 \mathrm{mM}$ IPTG.

\section{Supplementary Files}

This is a list of supplementary files associated with this preprint. Click to download.

- Supplementarymaterial.docx 